\title{
Measuring Human Perception of Biophilically-Driven Design with Facial Micro-expressions Analysis and EEG Biosensor
}

\author{
Andrea Macruz ${ }^{1(\bowtie)}$, Ernesto Bueno ${ }^{2}$, Gustavo G. Palma ${ }^{3}$, Jaime Vega ${ }^{4}$, \\ Ricardo A. Palmieri ${ }^{5}$, and Tan Chen $\mathrm{Wu}^{6}$ \\ 1 Tongji University, Shanghai, China \\ andrea.macruz@uol.com.br \\ 2 Universidade Presbiteriana Mackenzie, Sao Paulo, Brazil \\ 3 Centro Universitário Belas Artes, Sao Paulo, Brazil \\ ${ }^{4}$ Universidade Anhembi Morumbi, Sao Paulo, Brazil \\ 5 Universidade Federal do ABC, Santo André, Brazil \\ 6 Instituto do Coração - InCor - HCFMUSP, Sao Paulo, Brazil
}

\begin{abstract}
This paper investigates the role technology and neuroscience play in aiding the design process and making meaningful connections between people and nature. Using two workshops as a vehicle, the team introduced advanced technologies and Quantified Self practices that allowed people to use neural data and pattern recognition as feedback for the design process. The objective is to find clues to natural elements of human perception that can inform the design to meet goals for well-being. A pattern network of geometric shapes that achieve a higher level of monitored meditation levels and point toward a positive emotional valence is proposed. By referencing biological forms found in nature, the workshops utilized an algorithmic process that explored how nature can influence architecture. To measure the impact, the team used FaceOSC for capture and an Artificial Neural Network for micro-expression recognition, and a MindWave sensor manufactured by NeuroSky, which documented the human response further. The methodology allowed us to establish a boundary logic, ranking geometric shapes that suggested positive emotions and a higher level of monitored meditation levels. The results pointed us to a deeper level of understanding relative to geometric shapes in design. They indicate a new way to predict how well-being factors can clarify and rationalize a more intuitive design process inspired by nature.
\end{abstract}

Keywords: Algorithmic design · Neuroscience $\cdot$ AI $\cdot$ Biosensor $\cdot$ Biophilia

\section{Introduction}

Nature has been guiding our behavior, from 2-million-years in outdoor environments. From the past 12,000 years on, with the appearance of Homo sapiens, we started producing food, changing our surroundings, and building shelters, indoor environments [11]. 
However, currently, we spend ninety percent of our time in indoor environments [22], so there is a discrepancy between the type of spaces we are used to experiencing in the past and the ones we are experiencing now. So, what is the impact of that on our bodies and minds? Suppose the environment is a source of information that guides our behavior, such as temperature, light, and seasonal changes. What kind of information are we managing to extract from the spaces that we are creating? With the contemporary advances in neuroscience, advanced technologies, and Quantified Self (QS) practices, is it possible to further understand how our current interactions with our built environment might be affected by the natural setting?

This paper investigates the role technology and neuroscience play in aiding the design process and making meaningful connections between people and nature. As of the date of this paper, two workshops were used as a vehicle, adding 47 participants, $57 \%$ men and $43 \%$ women. The average age of the participants was 23 years old, with a minimum age of 19 and a maximum of 42, composed of undergraduate and graduate students of architecture and design. During the workshop, the team introduced advanced technologies and QS practices that allowed the use of neural data and pattern recognition as feedback for the design process. The objective is to find clues to natural elements of human perception that can inform the design to meet goals for well-being. The means developed toward this objective include proposing a pattern network of geometric shapes that achieve a higher level of monitored meditation levels [10] and point toward a positive emotional valence [18]. By referencing biological forms found in nature, the workshops utilized an algorithmic process that explored how nature can influence architecture and design.

To measure the impact, the team used interfaces designed for this purpose based on low-coast technology and open-source software, such as FaceOSC and Processing, which documented the human response further. The participants started by creating geometries inspired by nature through algorithmic modeling; this allowed specific parameters to be enforced (further details presented from Sect. 3.1 and onwards). The projects that were most aligned with the biophilic design were compiled into a seven-minute presentation, which each participant watched while recorded a video-selfie. An inter-individual analysis was made to classify the emotional valences provoked by each instance. The participants' facial expressions were then analyzed and ranked using FaceOSC and an Artificial Neural Network (ANN) for Gesture Recognition [5]. This process was trained to correlate with micro-expressions using Geneva Affective Picture and Database [7]. In parallel, an intra-individual analysis was used, and the designs were tested with a commercial electroencephalography (EEG) sensor. The same designs were then observed one more time to estimate brain modulations in the observer's attention and meditation levels (AT, MT). These datasets were used to redesign the initial drawings based upon the degree of positive emotional valence.

Upon review of the drawings as evidence, the low-cost interface (FaceOSC and Max MSP) and MindWave (explained in Sects. 3.2 and 3.3 of this paper) allowed us to establish a boundary logic, ranking geometric shapes that pointed to positive emotions and a higher level of monitored meditation levels. The results pointed us to a deeper level of understanding relative to geometric shapes in design. They indicate a new way to predict how well-being factors can clarify and rationalize a more intuitive design process inspired 
by nature. This investigation seeks a better qualitative understanding of the fundamental preferences we all share, densely overlaid with individual and cultural interactions that add endless complexities and variations. It does not intend to be universally applicable; these are initial steps towards developing designs that align with our preferences.

\section{Biophilic Design Framework}

The term biophilia was coined in 1964 by the psychologist and philosopher Erich Fromm, meaning "love of life" [8]. In 1984, it was diffused and published by biologist Edward O. Wilson [25]. The biophilia hypothesis suggests that humans have an innate tendency to seek connections with the natural world. Also, in that same year, Roger Ulrich released a landmark paper that established the healing power of nature in which he compares recovery rates of patients that have and do not have a view to nature [24]. In 1986, Gordon Orians and Judith Heerwagen posited the Savanna Hypothesis, defending that we should be genetically predisposed to prefer particular types and natural scenery, such as the savanna, due to our evolution in East Africa during the Pleistocene [16]. This hypothesis was a turning point for biophilia and much of the recent discoveries in that area. Evolutionary psychology and related research suggest that human beings prefer colors that refer to the savannah, especially colors found in healthy vegetation such as blue, green, and earth tones. Colors commonly found in healthy natural landscapes indicate clean water, nutrient-rich vegetation, fruits, and flowers.

In 1993, Stephen Kellert and Edward Wilson published the book The Biophilia Hypothesis [13]. According to them, the mid-range fractal dimension of a savannah landscape provides survival advantages such as the effortless conveyance of basic structural information environments with higher fractal dimensions, such as forest, can hide predators and thus present more danger. In contrast, environments with much lower fractal dimensions are too open and too exposed to offer protection and food sources. That is why humans have an innate preference for those kinds of environments, leading to natural comfort and well-being.

The transition of the biophilia hypothesis to biophilic design was the topic of a 2004 conference on the built environment and a latter book on biophilic design, called Biophilic Design: The Theory, Science, and Practice of Bringing Buildings to Life [12]. Biophilic design is based on the biophilia hypothesis. It encourages natural systems and features to create built environments, taking advantage of nature's affinity to create spaces where we can improve people's physical and well-being.

In 2014, Terrapin Bright Green released The 14 Patterns of Biophilic Design: Improving Health and Well-Being in the Built Environment [9]. It recently evolved in 2015 to five principles and 24 strategies with the manual The Practice of Biophilic Design, by Stephen Kellert and Elizabeth Calabrese [11]. Recently, many other architects, mathematicians, neuroscientists, and psychologists have been revising the theories and adding significant contributions to biophilic design. Relative to the geometrical approach, analysis and composition of architectural forms, Nikos Salingaros' [21] and Omid Kardan's [6] papers are very relevant, along with the books from Christopher Alexander [1] and from Sussman and Hollander [23]. In Cognitive Architecture: Designing for How We Respond to the Built Environment, Sussman and Hollander show how people are visually oriented and why they prefer a bilateral symmetrical form due to biology [23]. 
The biophilic design parameters used for the development of the workshops' projects, based on [3] and the references above, were:

1. mid-range fractal dimension

2. symmetries

3. growing formulas, such as the Fibonacci sequence and harmonic curves

4. focal point

5. complementary contrasts such as the use of lines mixed with curves

6. soft colors or white

The works created in the workshops are not Biophilic Design samples since this involves the design alignment with most of the principles and strategies related to biophilic design. Just the parameters mentioned above were chosen because they relate to vision, which is the most developed within all the human sensoria.

\section{Materials and Methods}

\subsection{Algorithmic Modeling of Biophilically-Driven Geometry}

Once the biophilic design concepts had been introduced and determined, the algorithmic modeling started. The workshops then focused on the modeling methods that had the most potential for applying these concepts and biophilic patterns. The modeling tools used were the NURBS modeling software Rhinoceros, with the graphical algorithms' editor Grasshopper and plug-ins, such as Mesh Edit, Mesh Tools, Pufferfish, and Weaverbird [19]. The time of the training stage was short, and the participants did not know how to program. Thus, it was decided not to work with code programming routines and to discard the use of other algorithmic plug-ins that would otherwise be relevant, such as Anemone [9].

The participants were instructed to recreate the shapes of the geometric basis of the biological system chosen by them. The similarities and differences of organic forms with NURBS surfaces were considered, and their attributes to define strategies for subdividing and populating surfaces [4] were discussed. Congruently, the focus was on constructing surfaces by edge curves combined with extrusion and loft operations [4].

Algorithmically, it was demonstrated how to define transformations in sequence, forming hierarchical sets or progressive growth, by setting lists of transformation vectors. Unlike precedents [9], the generative growth was presented with examples that exhibit a recursive logic without the need for loops or recursive functions. For this, the golden ratio was used for incremental scale and rotation, and the Fibonacci sequence was used as a variable for the translation. This was possible due to the recursive nature of these mathematical constants, which have been found in patterns in nature [15] (Fig. 1). Another transformation relevant to biophilia was the Mirror Cut function, supported by Pufferfish. This function allows composing a symmetrical shape out of any shape by joining it with the mirrored part, merging the joints. It is advantageous with polygon meshes due to its efficiency in both computation and visualization. 
Concerns about efficiency are relevant to visualize complex shapes through fast rendering and augmented reality in the following experiments. For this, ways of discretizing NURBS surfaces as meshes were taught, as well as how to and apply the interpolation necessary to create base shapes with continuous and organic curvatures. Taking advantage of the work with these meshes, subdivision and smoothing functions were added (such as the Catmull-Clark and the Charles Loop algorithms implemented by Piacentino in Weaverbird [19]), and also, maelstrom deformations and mesh face reductions by Boolean patterns of repetition and pseudo-random sorted values. Adaptations of one algorithmic definition to different base geometries were demonstrated to promote design alternatives [17]. Next, the definitions were shared with the participants to build upon these or use as a reference for developing their own design ideas and applying productive creativity [9]. Later, the participants were directed and advised in the development of their proposals.
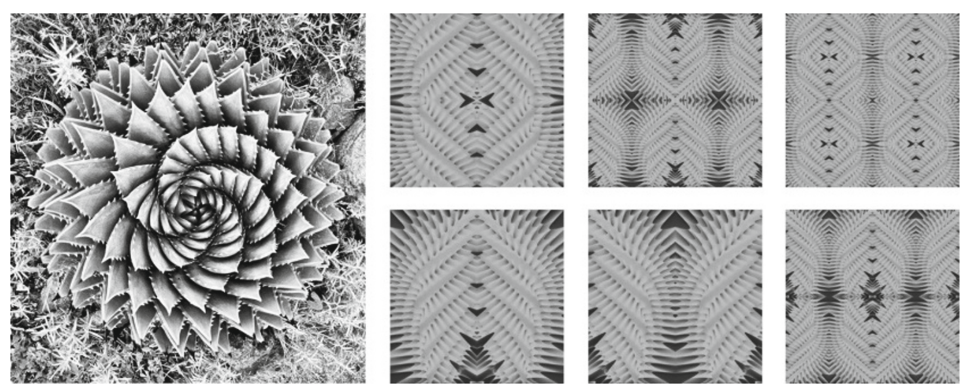

Fig. 1. Students' work: natural reference and geometric constructions of natural patterns

\subsection{Facial Micro-expressions Analysis}

This part of the methodology was based on an inter-individual analysis to classify the emotional valences provoked by the projects. From the geometries inspired by nature created by the participants, the projects that were most aligned with biophilic design were selected and compiled into a seven-minute presentation. Each participant recorded a video-selfie while watching this compilation to be used as a source for reading their facial micro-expressions. These expressions were registered using open-source software FaceOSC, from which the data was sent to an interface that uses an Artificial Neural Network (ANN) implemented on Max MSP by IRL Labs, the Machine Learning Library for Gesture Recognition [5] (Fig. 2). Based on a previous version, the Interface for Capturing and Identifying States of Poetic Presences (ICISPP), that classifies states of Poetic Presence in performing arts [18], this interface correlates facial expressions to emotional responses found in the participant's video-selfies. For this purpose, the ANN was trained using an actor's facial expression in reaction to the Geneva Affective Picture and Database - GAPED [7]. This database was designed to trigger the viewer's emotional responses according to three emotional valences: Neutral, Negative, and Positive [7]. Therefore, the interface can correlate participant's expressions according to each 
emotional valence category caused by each project using their video-selfies. It delivers graphics that allow us to infer subjective relations, correlating that seven-minute presentation to the intensity of the valences through time. Subsequently, a discussion was established about specific aspects of these projects (lines, shapes, perimetry, surface patterns, depths, symmetries, the focus of attention, the overall organization of the gaze, and others), which may have relations with the state indexes and classification of valences raised before.

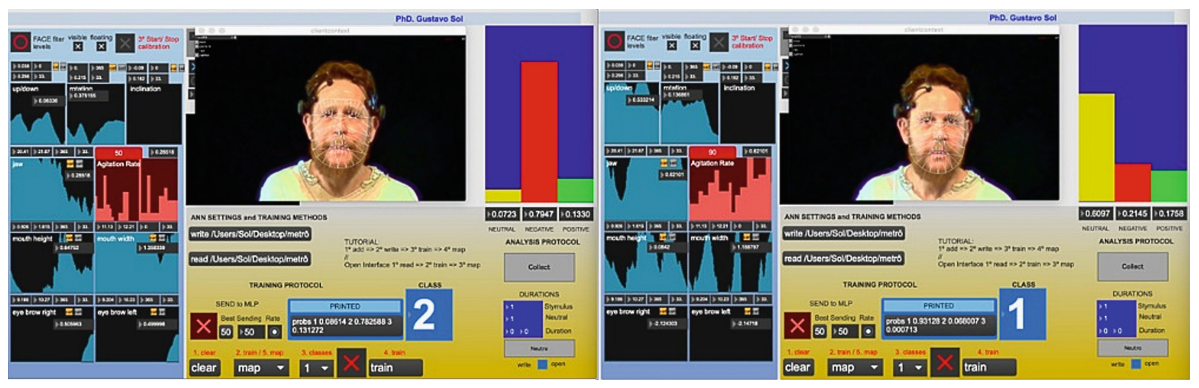

Fig. 2. Screen captures of the ICISPP setup with an ANN trained for micro-expressions analysis of recorded video-selfie at two different moments

\subsection{EEG Biosensor and Customized Data Capture Tool}

In addition to the above, an intra-individual analysis was used to detect the levels of attention required by each project. Participants who never saw the projects watched this seven-minute presentation, and their neural states were collected using a commercial EEG: the MindWave biosensor, manufactured by NeuroSky [14]. First, a digital toolset was built to collect data from the brainwave sensor [10] and converted these numbers in graphics to further analysis and comparisons with data captured from other devices and biofeedback techniques developed by our team. The idea of this application came from the simplicity and flexibility in making code using the open-source development interface called Processing [20]. With this technological strategy, it was designed a process using this workflow to deploy the toolset: NeuroSky MindWave [10, 14] $\rightarrow$ ThinkGear Connector $\rightarrow$ TCP/IP commands $\rightarrow$ ThinkGear Java socket [2] $\rightarrow$ Processing 2.2.1 $[20] \rightarrow$ Timer $\rightarrow$ Suggested Image $\rightarrow$ generate a TXT file with all the data collected at the end of the process.

With this customization developed using Processing IDE [20] as the main management tool, it was possible to visualize the workshop's images. Simultaneously, the software recorded the biosensor data generated, writing it in a text file. With a configurable timer to determine the amount of time of exposure that the users could have over the analyzed image, the MindWave sensor collected and filtered four different kinds of data from the ThinkGear Java Socket [2]: Attention Level, Meditation Level, Poor Signal Quality, and EEG Events (Delta, Theta, Low Alpha, High Alpha, Low Beta, High Beta, Low Gamma, and Mid Gamma Waves). 


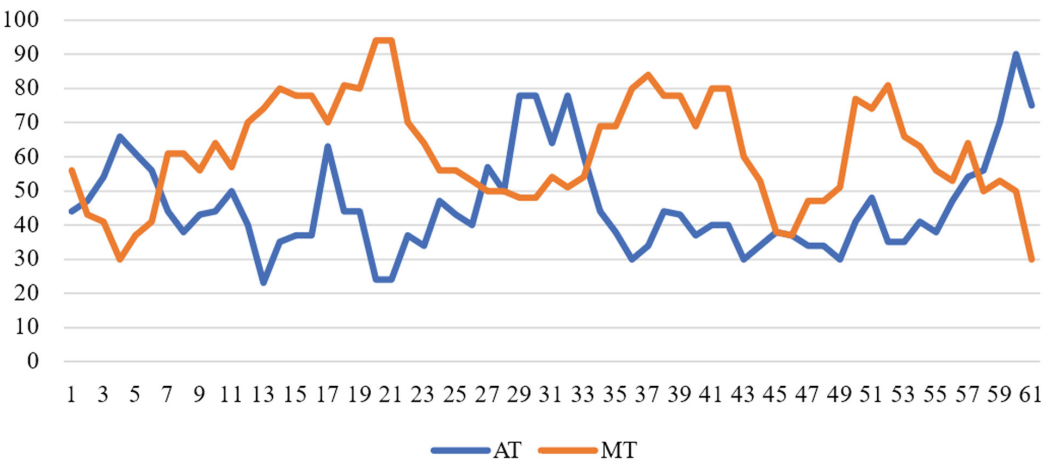

Fig. 3. Graphic plot for the readings of average attention levels (AT) and meditation levels (MT) from all the perceptions of one of the projects from workshop 1 (showed at Fig. 4, upper right) in $60 \mathrm{~s}$

In addition, the toolset was able to collect potential interference or signal noise. By detecting the level of these signals emitted by the hardware, the software allowed, through its interface, to check if the communication is full, noisy, or null. Finishing this data collection process, generated during the image visualization, it was possible to extract the data using a spreadsheet software that allowed creating tables and graphics, like the one in Fig. 3. These graphics were necessary to understand and compare all the numbers collected by the application.

\section{Quantified Self Practice Results}

The inter and intra-individual analyses were compared, verifying possible patterns in the degrees of attentional excitement, relaxation, and valences that could be associated with the projects. In the first workshop, it was observed that the results that pointed to positive emotions and a higher level of monitored meditation levels were the ones that presented symmetric shapes, fractal geometries, and soft colors, blue or green (Fig. 4). In the second workshop were the ones that had circular forms, growing sequences, or harmonic, continued curves (Fig. 5). It was observed that designs that had fewer curves or that the curves were discontinued had significantly lower meditation levels. 

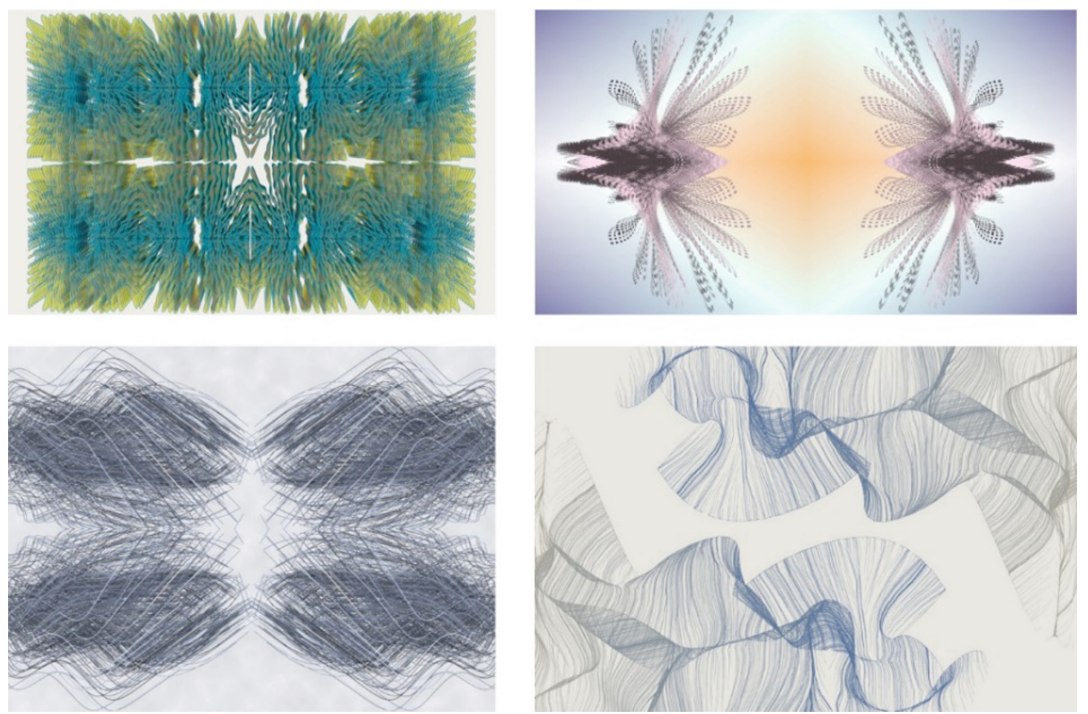

Fig. 4. Designs that achieved a higher level of monitored meditation levels and pointed towards a positive emotional valence on workshop 1
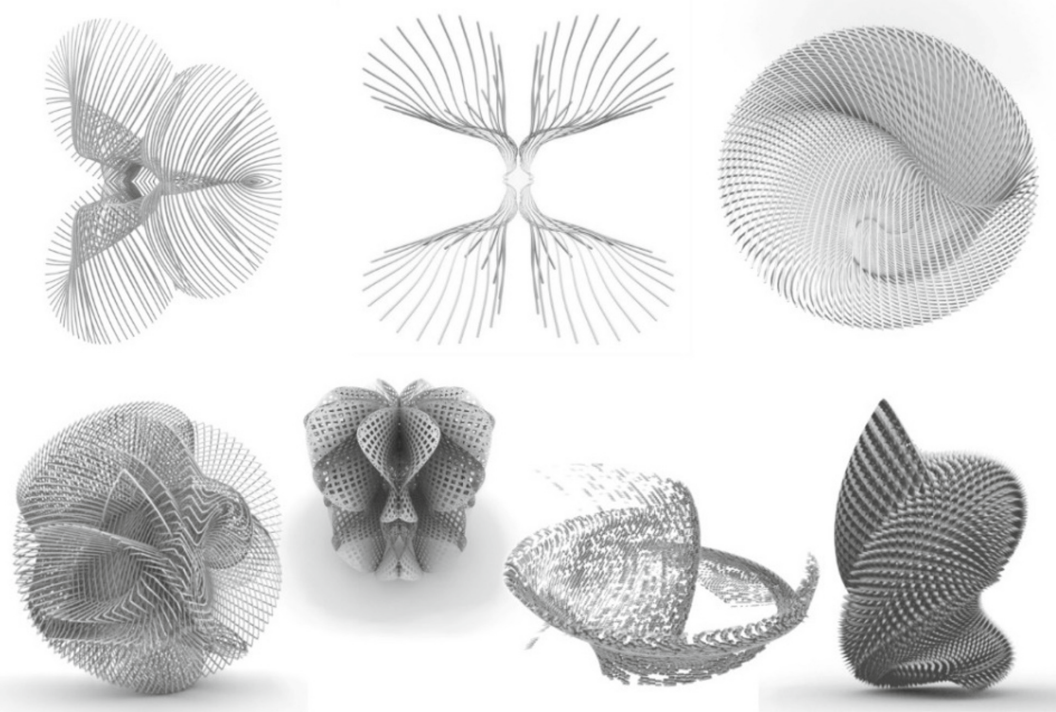

Fig. 5. Designs that achieved a higher level of monitored meditation levels and pointed towards a positive emotional valence on workshop 2

\section{Conclusions}

This research explores the potential that technology and neuroscience have in helping the design process and making meaningful connections between people and nature. 
The team used two workshops as a vehicle to incorporate experimental technology and QS practices, which enabled people to use neural data and pattern recognition as design feedback. It started with research in biophilic design and employed algorithmic modeling software to develop the projects, resulting in bidimensional images. The projects that were most aligned with biophilic design were tested with two methodological strategies, inter and intra-individual analyses. The first one used the IICPP instrumentation to acquire and analyze facial micro-expressions, and the second one used a customized EEG data workflow to verify the intensities of brainwaves. Their results were divided into two categories: emotions valence and arousal and level of monitored meditation. It was important to have both analyses working together to cross data and better understand the human feedback towards the drawings. These specific toolsets and methods were chosen because they are both low-cost and accessible during the workshops. These outputs were used to rethink the designs that had been produced.

The objective was to inform the design for well-being by identifying clues of natural elements of human perception. For this, a pattern network of geometric shapes that point toward high visual quality and appealing features was proposed. By referencing biological forms and conditions found in nature, the works utilized an algorithmic process to explore how these attributes can be applied to architecture and design. Upon review of the drawings as evidence, the two methodological strategies mentioned above allowed us to establish a boundary logic, ranking geometric shapes that pointed to positive emotions and a higher level of monitored meditation levels. The results pointed us to a deeper level of understanding relative to the perception of geometric shapes in design. They indicate that our surroundings' characteristics, natural and artificial, may bear to some of our innate behaviors in the natural settings and point to a new way to predict how well-being factors can clarify and rationalize a more intuitive design process. This paper can also be used as a reflection on how designers in the age of AI may use science to design spaces that help us develop humanistic traits and abilities to enhance the built environment.

What we have learned offers an input relative to using biological and emotional data as feedback to support a more heightened experience of the relationship between humans and nature. Although there is much work to be done and this paper only documents two sample series of projects, the methodology allowed us to begin to predict and forecast how our design could respond to human interaction. The use of algorithmic design allowed us to create complex drawings. The use of a biosensor and a customized interface for facial micro-expressions analysis allowed us to understand biological feedback and to synthesize geometries that trigger an autonomic response. For the future, continued research will grow our analyzed data for stronger conclusions, such as new methodological procedures that take the difference of individuals into consideration. The following steps might include augmented reality and interactive holographic experiences in expanded immersive spaces to test the morphology of the projects that move from the canvas to the physical space and its possible changes in perception.

\section{References}

1. Alexander, C.: The Nature of Order, Book 1: The Phenomenon of Life, 1st edn. Center for Environmental Structure, Berkeley (2002) 
2. Borg, A.: ThinkGear-Java-Socket. Available via GITHUB (2020). https://github.com/borg/ ThinkGear-Java-socket. Accessed 3 Jan 2021

3. Browning, W., Ryan, C., Clancy, J.: Patterns of biophilic design: improving health \& wellbeing in the built environment. Available via TERRAPIN (2014). www.terrapinbrightgreen. com/reports/14-patterns/. Accessed 20 Jan 2021

4. Bueno, E.: Algorithmic form generation of a radiolarian pavilion. IJAC (2008). https://doi. org/10.1260/1478-0771.7.4.677

5. Bullock, J., Momeni, A.: ml.lib: Robust, cross-platform, open-source machine learning for max and pure data. Available via ALI MOMENI (2015). http://alimomeni.net/wordpress/wpcontent/uploads/2015/05/NIME-2015-ml.lib-Momeni-Bullock.pdf. Accessed 4 Jan 2021

6. Coburn, A., et al.: Psychological responses to natural patterns in architecture. J. Environ. Psychol. (2019). https://doi.org/10.1126/science.6143402

7. Dan-Glauser, E.S., Scherer, K.R.: The Geneva Affective Picture Database (GAPED): a new 730 picture database focusing on valence and normative significance. Behav. Res. (2011). https://doi.org/10.3758/s13428-011-0064-1

8. Fromm, E.: The Heart of Man, 1st edn. Harper \& Row, New York (1964)

9. Henriques, G.C., Bueno, E., Lenz, D., Sardenberg, V.: Generative systems: intertwining physical, digital and biological processes, a case study. In: 37th eCAADe \& 23rd SIGraDi, vol. 1, pp. 25-34 (2019). http://papers.cumincad.org/cgi-bin/works/paper/ecaadesigradi2019_100

10. Katona, J., Farkas, I., Ujbanyi, T., Dukan, P., Kovari, A.: Evaluation of the NeuroSky MindFlex EEG headset brain waves data. IEEE (2014). https://doi.org/10.1109/SAMI.2014.6822382

11. Kellert, S.R., Calabrese, E.: The practice of biophilic design. Available via BIOPHILIC DESIGN (2015). www.biophilic-design.com. Accessed 20 Jan 2021

12. Kellert, S.R., Heerwagen, J.H., Mador, M.: Biophilic Design: The Theory, Science and Practice of Bringing Buildings to Life, 1st edn. Wiley, Hoboken (2008)

13. Kellert, S.R., Wilson, E.O.: The Biophilia Hypothesis, 1st edn. Island Press, Washington, D.C. (1993)

14. NeuroSky: EEG: the ultimate guide. Available via NEUROSKY (n.d.). http://neurosky.com/ biosensors/eeg-sensor/ultimate-guide-to-eeg/. Accessed 3 Jan 2021

15. Omotehinwa, T.O., Ramon, S.O.: Fibonacci numbers and golden ratio in mathematics and science. IJCIT 2(4) (2013). www.ijcit.com/archives/volume2/issue4/Paper020414.pdf

16. Orians, G.H., Heerwagen, J.H.: Evolved responses to landscapes. In; Barkow, J.H., Cosmides, L., Tooby, J. (eds.) The Adapted Mind: Evolutionary Psychology and the Generation of Culture, 1st edn. Oxford University Press, New York (1992)

17. Oxman, R., Gu, N.: Theories and models of parametric design thinking. In: 33rd eCAADe, vol. 2, pp. 477-482 (2015). http://papers.cumincad.org/cgi-bin/works/paper/ecaade2015_33

18. Palma, G.G.: Estados de presenças poéticas mapeadas pela técnica de Eletroencefalografia (EEG) e pela frequência cardíaca (BPM) e uma proposta de criação performativa por meio do sensoriamento neurofisiológico ao vivo. Ph.D. thesis, ECA-USP, São Paulo (2017)

19. Piacentino, G.: Weaverbird: topological mesh editing for architects. Arch. Des. (2013). https:// doi.org/10.1002/ad.1571

20. Processing: Available via PROCESSING (n.d.). https://processing.org/. Accessed 10 Jan 2021

21. Salingaros, N.A.: Adaptive versus random complexity. New Des. Ideas 2(2), 51-61 (2018)

22. Sturgeon, A.: Creating Biophilic Buildings, 1st edn. Ecotone Publishing, Canada (2017)

23. Sussman, A., Hollander, J.B.: Cognitive Architecture: Designing for How We Respond to the Built Environment, 1st edn. Routledge, New York (2014)

24. Ulrich, R.S.: View through a window may influence recovery from surgery. Science (1984). https://doi.org/10.1126/science.6143402

25. Wilson, E.O.: Biophilia, 1st edn. Harvard University Press, Cambridge (1984) 
Open Access. This chapter is licensed under the terms of the Creative Commons Attribution 4.0 International License (http://creativecommons.org/licenses/by/4.0/), which permits use, sharing, adaptation, distribution and reproduction in any medium or format, as long as you give appropriate credit to the original author(s) and the source, provide a link to the Creative Commons license and indicate if changes were made.

The images or other third party material in this chapter are included in the chapter's Creative Commons license, unless indicated otherwise in a credit line to the material. If material is not included in the chapter's Creative Commons license and your intended use is not permitted by statutory regulation or exceeds the permitted use, you will need to obtain permission directly from the copyright holder.

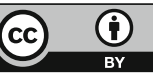

